1 Hacettepe Journal of Mathematics and Statistics

$\bigcap$ Volume $44(5)$ (2015), $1099-1108$

\title{
Signed degree sequences in signed multipartite graphs
}

\author{
S. Pirzada* and T. A. Naikoo ${ }^{\dagger}$
}

\begin{abstract}
A signed $k$-partite graph (signed multipartite graph) is a $k$-partite graph in which each edge is assigned a positive or a negative sign. If $G\left(V_{1}, V_{2}, \cdots, V_{k}\right)$ is a signed $k$-partite graph with $V_{i}=$ $\left\{v_{i 1}, v_{i 2}, \cdots, v_{i n_{i}}\right\}, 1 \leq i \leq k$, the signed degree of $v_{i j}$ is $\operatorname{sdeg}\left(v_{i j}\right)=$ $d_{i j}=d_{i j}^{+}-d_{i j}^{-}$, where $1 \leq i \leq k, 1 \leq j \leq n_{i}$ and $d_{i j}^{+}\left(d_{i j}^{-}\right)$is the number of positive (negative) edges incident with $v_{i j}$. The sequences $\alpha_{i}=\left[d_{i 1}, d_{i 2}, \cdots, d_{i n_{i}}\right], 1 \leq i \leq k$, are called the signed degree sequences of $G\left(V_{1}, V_{2}, \cdots, V_{k}\right)$. The set of distinct signed degrees of the vertices in a signed $k$-partite graph $G\left(V_{1}, V_{2}, \cdots, V_{k}\right)$ is called its signed degree set. In this paper, we characterize signed degree sequences of signed $k$-partite graphs. Also, we give the existence of signed $k$-partite graphs with given signed degree sets.
\end{abstract}

Keywords: Signed graphs, signed multipartite graph, signed degree, signed set. 2000 AMS Classification: $05 \mathrm{C} 22$.

Received 17/09/2011 : Accepted 24/06/2014 Doi : 10.15672/HJMS.2015449661

\section{Introduction}

A signed graph is a graph in which each edge is assigned a positive or a negative sign. The concept of signed graphs is given by Harary [3]. Let $G$ be a signed graph with vertex set $V=\left\{v_{1}, v_{2}, \cdots, v_{n}\right\}$. The signed degree of $v_{i}$ is $\operatorname{sdeg}\left(v_{i}\right)=d_{i}=d_{i}^{+}-d_{i}^{-}$, where $1 \leq i \leq n$ and $d_{i}^{+}\left(d_{i}^{-}\right)$is the number of positive(negative) edges incident with $v_{i}$. A signed degree sequence $\sigma=\left[d_{1}, d_{2}, \cdots, d_{n}\right]$ of a signed graph $G$ is formed by listing the vertex signed degrees in non-increasing order. An integral sequence is $s$-graphical if it is the signed degree sequence of a signed graph. Also, a non-zero sequence $\sigma=\left[d_{1}, d_{2}, \cdots, d_{n}\right]$ is a standard sequence if $\sigma$ is non-increasing, $\sum_{i=1}^{n} d_{i}$ is even, $d_{1}>0$, each $\left|d_{i}\right|<n$ and

\footnotetext{
*Department of Mathematics, University of Kashmir, Srinagar, Kashmir, India Email: sdpirzada@yahoo.co.in; pirzadasd@kashmiruniversity.ac.in

${ }^{\dagger}$ Department of Mathematics, Islamia College for Science and Commerce, Srinagar, Kashmir, India

Email:tariqnaikoo@yahoo.co.in
} 
$\left|d_{1}\right| \geq\left|d_{n}\right|$

The following result, due to Charttrand et al. [1], gives a necessary and sufficient condition for an integral sequence to be $s$-graphical, and this is similar to Hakimi's result for degree sequences in graphs [2].

Theorem 1. A standard integral sequence $\sigma=\left[d_{1}, d_{2}, \cdots, d_{n}\right]$ is $s$-graphical if and only if

$$
\sigma^{\prime}=\left[d_{2}-1, d_{3}-1, \cdots, d_{d_{1}+s+1}-1, d_{d_{1}+s+2}, \cdots, d_{n-s}, d_{n-s+1}+1, \cdots, d_{n}+1\right]
$$

is $s$-graphical for some $s, 0 \leq s \leq \frac{n-1-d_{1}}{2}$.

The next result [12] provides a good candidate for parameter $s$ in Theorem 1 .

Theorem 2. A standard integral sequence $\sigma=\left[d_{1}, d_{2}, \cdots, d_{n}\right]$ is s-graphical if and only if

$$
\sigma_{m}^{\prime}=\left[d_{2}-1, d_{3}-1, \cdots, d_{d_{1}+m+1}-1, d_{d_{1}+m+2}, \cdots, d_{n-m}, d_{n-m+1}+1, \cdots, d_{n}+1\right]
$$

is $s$-graphical, where $m$ is the maximum non-negative integer such that $d_{d_{1}+m+1}>$ $d_{n-m+1}$.

The set of distinct signed degrees of the vertices in a signed graph $G$ is called its signed degree set. In [6], it is proved that every set of positive (negative) integers is the signed degree set of some connected signed graph and the smallest possible order for such a signed graph is also determined. Hoffman and Jordan [4] have shown that the degree sequences of signed graphs can be characterized by a system of linear inequalities. The set of all $n$-tuples satisfying this system of linear inequalities is a polytope $P_{n}$. In [5], Jordan et al. have proved that $P_{n}$ is the convex hull of the set of degree sequences of signed graphs of order $n$. We can find more results on signed degrees in [4,5].

A signed bipartite graph is a bipartite graph in which each edge is assigned a positive or a negative sign. Let $G(U, V)$ be a signed bipartite graph with $U=\left\{u_{1}, u_{2}, \cdots, u_{p}\right\}$ and $V=\left\{v_{1}, v_{2}, \cdots, v_{q}\right\}$. Then signed degree of $u_{i}$ is $\operatorname{sdeg}\left(u_{i}\right)=d_{i}=d_{i}^{+}-d_{i}^{-}$, where $1 \leq i \leq p$ and $d_{i}^{+}\left(d_{i}^{-}\right)$is the number of positive (negative) edges incident with $u_{i}$ and signed degree of $v_{j}$ is $\operatorname{sdeg}\left(v_{j}\right)=e_{j}=e_{j}^{+}-e_{j}^{-}$, where $1 \leq j \leq q$ and $e_{j}^{+}\left(e_{j}^{-}\right)$is the number of positive (negative) edges incident with $v_{j}$. The sequences $\alpha=\left[d_{1}, d_{2}, \cdots, d_{p}\right]$ and $\beta=\left[e_{1}, e_{2}, \cdots, e_{q}\right]$ are called the signed degree sequences of the signed bipartite graph $G(U, V)$. Two sequences $\alpha=\left[d_{1}, d_{2}, \cdots, d_{p}\right]$ and $\beta=\left[e_{1}, e_{2}, \cdots, e_{q}\right]$ are standard sequences if $\alpha$ is non-zero and non-increasing, $\left|d_{1}\right| \geq\left|d_{p}\right|, \sum_{i=1}^{p} d_{i}=\sum_{j=1}^{q} e_{j}, d_{1}>0$, each $\left|d_{i}\right| \leq q$, each $\left|e_{j}\right| \leq p$ and $\left|e_{j}\right| \leq\left|d_{1}\right|$.

The following result due to Pirzada et al. [8], gives necessary and sufficient conditions for two sequences of integers to be the signed degree sequences of some signed bipartite graph. .

Theorem 3. Let $\alpha=\left[d_{1}, d_{2}, \cdots, d_{p}\right]$ and $\beta=\left[e_{1}, e_{2}, \cdots, e_{q}\right]$ be standard sequences. Then, $\alpha$ and $\beta$ are the signed degree sequences of a signed bipartite graph if and only if there exist integers $r$ and $s$ with $d_{1}=r-s$ and $0 \leq s \leq \frac{q-d_{1}}{2}$ such that $\alpha^{\prime}$ and $\beta^{\prime}$ are the signed degree sequences of a signed bipartite graph, where $\alpha^{\prime}$ is obtained from $\alpha$ by deleting $d_{1}$ and $\beta^{\prime}$ is obtained from $\beta$ by reducing $r$ greatest entries of $\beta$ by 1 each and adding $s$ least entries of $\beta$ by 1 each. 
The set of distinct signed degrees of the vertices in a signed bipartite graph $G(U, V)$ is called its signed degree set. The work for signed degree sets in signed bipartite graphs can be found in [7]. Also the work on signed degrees in signed tripartite graphs can be found in $[10,11]$.

\section{Signed degree sequences in signed $k$-partite graphs}

A signed $k$-partite graph (signed multipartite graph) is a $k$-partite graph in which each edge is assigned a positive or a negative sign. Let $G\left(V_{1}, V_{2}, \cdots, V_{k}\right)$ be a signed $k$-partite graph with $V_{i}=\left\{v_{i 1}, v_{i 2}, \cdots, v_{i n_{i}}\right\}, 1 \leq i \leq k$. The signed degree of $v_{i j}$ is $\operatorname{sdeg}\left(v_{i j}\right)=d_{i j}=d_{i j}^{+}-d_{i j}^{-}$, where $1 \leq i \leq k, 1 \leq j \leq n_{i}$ and $d_{i j}^{+}\left(d_{i j}^{-}\right)$is the number of positive (negative) edges incident with $v_{i j}$. The sequences $\alpha_{i}=\left[d_{i 1}, d_{i 2}, \cdots, d_{i n_{i}}\right]$, $1 \leq i \leq k$, are called the signed degree sequences of $G\left(V_{1}, V_{2}, \cdots, V_{k}\right)$. Also the sequences $\alpha_{i}=\left[d_{i 1}, d_{i 2}, \cdots, d_{i n_{i}}\right], 1 \leq i \leq k$, of integers are $s$-graphical if $\alpha_{i}^{\prime} s$ are the signed degree sequences of some signed $k$-partite graph. Denote a positive edge $x y$ by $x y^{+}$and a negative edge $x y$ by $x y^{-}$. Several results on signed degree sequences in signed multipartite graphs can be found in [9]. We start with the following observation.

Theorem 4. Let $G\left(V_{1}, V_{2}, \cdots, V_{k}\right)$ be a signed $k$-partite graph with $V_{i}=\left\{v_{i 1}, v_{i 2}, \cdots, v_{i n_{i}}\right\}$, $1 \leq i \leq k$ and having $q$ edges. Then

$$
p=\sum_{i=1}^{k} \sum_{j=1}^{n_{i}} s \operatorname{deg}\left(v_{i j}\right) \equiv 2 q(\bmod 4)
$$

and the number of positive edges and negative edges of $G\left(V_{1}, V_{2}, \cdots, V_{k}\right)$ are respectively $\frac{2 q+p}{4}$ and $\frac{2 q-p}{4}$.

Proof. Let $v_{i j}\left(1 \leq i \leq k, 1 \leq j \leq n_{i}\right)$ be incident with $d_{i j}^{+}$positive edges and $d_{i j}^{-}$ negative edges so that

$$
\operatorname{sdeg}\left(v_{i j}\right)=d_{i j}^{+}-d_{i j}^{-} \text {while } \operatorname{deg}\left(v_{i j}\right)=d_{i j}^{+}+d_{i j}^{-} .
$$

Obviously, $\sum_{i=1}^{k} \sum_{j=1}^{n_{i}} \operatorname{deg}\left(v_{i j}\right)=2 q$.

Let $G\left(V_{1}, V_{2}, \cdots, V_{k}\right)$ have $g$ positive edges and $h$ negative edges. Then $q=g+h$,

$$
\sum_{i=1}^{k} \sum_{j=1}^{n_{i}} d_{i j}^{+}=2 g \text { and } \sum_{i=1}^{k} \sum_{j=1}^{n_{i}} d_{i j}^{-}=2 h .
$$

Further,

$$
\begin{aligned}
\sum_{i=1}^{k} \sum_{j=1}^{n_{i}} s \operatorname{deg}\left(v_{i j}\right) & =\sum_{i=1}^{k} \sum_{j=1}^{n_{i}}\left(d_{i j}^{+}-d_{i j}^{-}\right) \\
& =\sum_{i=1}^{k} \sum_{j=1}^{n_{i}} d_{i j}^{+}-\sum_{i=1}^{k} \sum_{j=1}^{n_{i}} d_{i j}^{-} \\
& =2 g-2 h .
\end{aligned}
$$

Hence,

$$
\begin{aligned}
p=\sum_{i=1}^{k} \sum_{j=1}^{n_{i}} s \operatorname{deg}\left(v_{i j}\right) & \equiv 2 g-2 h \\
& =2(q-h)-2 h \\
& =2 q-4 h,
\end{aligned}
$$

so that $p \equiv 2 q(\bmod 4)$. Again, from $g+h=q$ and $2 g-2 h=p$, we have $g=\frac{2 q+p}{4}$ and $h=\frac{2 q-p}{4}$. 
Corollary 5. A necessary condition for the $k$ sequences $\alpha_{i}=\left[d_{i 1}, d_{i 2}, \cdots, d_{i n_{i}}\right], 1 \leq i \leq$ $k$, of integers to be $s$-graphical is that $\sum_{i=1}^{k} \sum_{j=1}^{n_{i}} d_{i j}$ is even.

A zero sequence is a finite sequence each term of which is 0 . Clearly, every $k$ finite zero sequences are the signed degree sequences of a signed $k$-partite graph. If $\beta=\left[a_{1}, a_{2}, \cdots, a_{n}\right]$ is a sequence of integers, then the negative of $\beta$ is the sequence $\beta=\left[-a_{1},-a_{2}, \cdots,-a_{n}\right]$.

The next result follows by interchanging positive edges with negative edges.

Theorem 6. The sequences $\alpha_{i}=\left[d_{i 1}, d_{i 2}, \cdots, d_{i n_{i}}\right], 1 \leq i \leq k$, are the signed degree sequences of some signed $k$-partite graph if and only if $-\alpha_{i}=\left[-d_{i 1},-d_{i 2}, \cdots,-d_{i n_{i}}\right]$ are the signed degree sequences of some signed $k$-partite graph.

Assume without loss of generality, that a non-zero sequence $\beta=\left[a_{1}, a_{2}, \cdots, a_{n}\right]$ is non-increasing and $\left|a_{1}\right| \geq\left|a_{n}\right|$, for we can always replace $\beta$ by $-\beta$ if necessary. The $k$ sequences of integers $\alpha_{i}=\left[d_{i 1}, d_{i 2}, \cdots, d_{i n_{i}}\right], 1 \leq i \leq k$, are said to be standard sequences if $\alpha_{1}$ is non-zero and non-increasing, $\sum_{i=1}^{k} \sum_{j=1}^{n_{i}} d_{i j}$ is even, $d_{11}>0$, each $\left|d_{i j}\right| \leq \sum_{r=1, r \neq i}^{k} n_{r}, 1 \leq i \leq k, 1 \leq j \leq n_{i},\left|d_{11}\right| \geq\left|d_{1 n_{1}}\right|$ and $\left|d_{11}\right| \geq\left|d_{i j}\right|$ for each $2 \leq i \leq k, 1 \leq j \leq n_{i}$.

A complete signed $k$-partite graph is a complete $k$-partite graph in which each edge is assigned a positive or a negative sign. The following result provides a useful recursive test whether the $k$ sequences of integers form the signed degree sequences of some complete signed $k$-partite graph.

Theorem 7. Let $\alpha_{i}=\left[d_{i 1}, d_{i 2}, \cdots, d_{i n_{i}}\right], 1 \leq i \leq k$, be standard sequences and let $r=\frac{1}{2}\left(d_{11}+\sum_{j=2}^{k} n_{j}\right)$. Let $\alpha_{1}^{\prime}$ be obtained from $\alpha_{1}$ by deleting $d_{11}$ and $\alpha_{2}^{\prime}, \alpha_{3}^{\prime}, \cdots, \alpha_{k}^{\prime}$ be obtained from $\alpha_{2}, \alpha_{3}, \cdots, \alpha_{k}$ by reducing $r$ greatest entries of $\alpha_{2}, \alpha_{3}, \cdots, \alpha_{k}$ by 1 each and adding remaining entries of $\alpha_{2}, \alpha_{3}, \cdots, \alpha_{k}$ by 1 each. Then $\alpha_{i}$ are the signed degree sequences of some complete signed $k$-partite graph if and only if $\alpha_{i}^{\prime}$ are also signed degree sequences of some complete signed $k$-partite graph, $1 \leq i \leq k$.

Proof. Let $G^{\prime}\left(V_{1}^{\prime}, V_{2}^{\prime}, \cdots, V_{k}^{\prime}\right)$ be a complete signed $k$-partite graph with signed degree sequences $\alpha_{i}^{\prime}, 1 \leq i \leq k$. Let $V_{1}^{\prime}=\left\{v_{12}, v_{13}, \cdots, v_{1 n_{1}}\right\}$ and $V_{i}^{\prime}=\left\{v_{i 1}, v_{i 2}, \cdots, v_{i n_{i}}\right\}$, $2 \leq i \leq k$. Then a complete signed $k$-partite graph with signed degree sequences $\alpha_{i}$, $1 \leq i \leq k$, can be obtained by adding a vertex $v_{11}$ to $V_{1}^{\prime}$ so that there are $r$ positive edges from $v_{11}$ to those $r$ vertices of $V_{2}^{\prime}, V_{3}^{\prime}, \cdots, V_{k}^{\prime}$, whose signed degrees were reduced by 1 in going from $\alpha_{i}$ to $\alpha_{i}^{\prime}$, and there are negative edges from $v_{11}$ to the remaining vertices of $V_{2}^{\prime}, V_{3}^{\prime}, \cdots, V_{k}^{\prime}$, whose signed degrees were increased by 1 in going from $\alpha_{i}$ to $\alpha_{i}^{\prime}$. Note that the signed degree of $v_{11}$ is $r-\left(\sum_{j=2}^{k} n_{j}-r\right)=2 r-\sum_{j=2}^{k} n_{j}=d_{11}$.

Conversely, let $\alpha_{i}, 1 \leq i \leq k$, be the signed degree sequences of a complete signed $k$-partite graph. Let the vertex sets of the complete signed $k$-partite graph be $V_{i}=$ $\left\{v_{i 1}, v_{i 2}, \cdots, v_{i n_{i}}\right\}$ such that $\operatorname{sdeg}\left(v_{i j}\right)=d_{i j}, 1 \leq i \leq k, 1 \leq j \leq n_{i}$.

Among all the complete signed $k$-partite graphs with $\alpha_{i}, 1 \leq i \leq k$, as the signed degree sequences, let $G\left(V_{1}, V_{2}, \cdots, V_{k}\right)$ be one with the property that the sum $S$ of the signed degrees of the vertices of $V_{2}, V_{3}, \cdots, V_{k}$ joined to $v_{11}$ by positive edges is maximum. Let $d_{11}^{+}$and $d_{11}^{-}$be respectively the number of positive edges and the number of negative edges incident with $v_{11}$. Then $\operatorname{sdeg}\left(v_{11}\right)=d_{11}=d_{11}^{+}-d_{11}^{-}, \operatorname{deg}\left(v_{11}\right)=$ $d_{11}^{+}+d_{11}^{-}=\sum_{j=2}^{k} n_{j}$, and hence $d_{11}^{+}=\frac{1}{2}\left(d_{11}+\sum_{j=2}^{k} n_{j}\right)=r$. Let $U$ be the set of $r$ 
vertices of $V_{2}, V_{2}, \cdots, V_{k}$ with highest signed degrees and let $W=\cup_{j=2}^{k} V_{j}-U$. We claim that $v_{11}$ must be joined by positive edges to the vertices of $U$. If this is not true, then there exist vertices $v_{g h} \in U$ and $v_{i j} \in W$ such that the edge $v_{11} v_{g h}$ is negative and the edge $v_{11} v_{i j}$ is positive. Since $\operatorname{sdeg}\left(v_{g h}\right) \geq \operatorname{sdeg}\left(v_{i j}\right)$, there exist vertices $v_{m n}$ and $v_{p q}$ such that the edge $v_{g h} v_{m n}$ is positive and the edge $v_{i j} v_{p q}$ is negative. If the edge $v_{g h} v_{p q}$ is positive, then change the signs of the edges $v_{11} v_{g h}, v_{g h} v_{p q}, v_{p q} v_{i j}, v_{i j} v_{11}$ so that the edges $v_{11} v_{g h}$ and $v_{p q} v_{i j}$ are positive and the edges $v_{11} v_{i j}$ and $v_{g h} v_{p q}$ are negative. But if the edge $v_{g h} v_{p q}$ is negative, then $\operatorname{sdeg}\left(v_{g h}\right)<s d e g\left(v_{i j}\right)$, which is a contradiction. The case when $v_{m n}=v_{p q}$ follows by the same argument as in above.

Hence we obtain a complete signed $k$-partite graph with signed degree sequences $\alpha_{i}$, $1 \leq i \leq k$, in which the sum of the signed degrees of the vertices of $V_{2}, V_{3}, \cdots, V_{k}$ joined to $v_{11}$ by positive edges exceeds $S$, a contradiction.

Thus we may assume that $v_{11}$ is joined by positive edges to the vertices of $U$ and by negative edges to the vertices of $W$. So $G\left(V_{1}, V_{2}, \cdots, V_{k}\right)-v_{11}$ is a complete signed $k$-partite graph with $\alpha_{i}^{\prime}, 1 \leq i \leq k$, as the signed degree sequences.

Theorem 7 provides an algorithm of checking whether the standard sequences $\alpha_{i}$, $1 \leq i \leq k$, are the signed degree sequences, and for constructing a corresponding complete signed $k$-partite graph. Suppose $\alpha_{i}=\left[d_{i 1}, d_{i 2}, \cdots, d_{i n_{i}}\right], 1 \leq i \leq k$, be the standard signed degree sequences of a complete signed $k$-partite graph with parts $V_{i}=\left\{v_{i 1}, v_{i 2}, \cdots, v_{i n_{i}}\right\}$. Deleting $d_{11}$ and reducing $r=\frac{1}{2}\left(d_{11}+\sum_{j=2}^{k} n_{j}\right)$ greatest entries of $\alpha_{2}, \alpha_{3}, \cdots, \alpha_{k}$ by 1 each and adding remaining entries of $\alpha_{2}, \alpha_{3}, \cdots, \alpha_{k}$ by 1 each to form $\alpha_{2}^{\prime}, \alpha_{3}^{\prime}, \cdots, \alpha_{k}^{\prime}$. Then edges are defined by $v_{11} v_{i j}^{+}$if $d_{i j}^{\prime} s$ are reduced by 1 and $v_{11} v_{i j}^{-}$if $d_{i j}^{\prime} s$ are increased by 1 . For $-\alpha_{i}, 1 \leq i \leq k$, edges are defined by $v_{11} v_{i j}^{-}$if $d_{i j}^{\prime} s$ are reduced by 1 and $v_{11} v_{i j}^{+}$if $d_{i j}^{\prime} s$ are increased by 1 . If the conditions of standard sequences do not hold, then we delete $d_{i 1}$ for that $i$ for which the conditions of standard sequences get satisfied. If this method is applied recursively, then a complete signed $k$-partite graph with signed degree sequences $\alpha_{i}, 1 \leq i \leq k$, is constructed.

The next result gives necessary and sufficient conditions for the $k$ sequences of integers to be the signed degree sequences of some signed $k$-partite graph.

Theorem 8. Let $\alpha_{i}=\left[d_{i 1}, d_{i 2}, \cdots, d_{i n_{i}}\right], 1 \leq i \leq k$, be standard sequences. Then $\alpha_{i}$, $1 \leq i \leq k$, are the signed degree sequences of a signed $k$-partite graph if and only if there exist integers $r$ and $s$ with $d_{11}=r-s$ and $0 \leq s \leq \frac{1}{2}\left(\sum_{j=2}^{k} n_{j}-d_{11}\right)$ such that $\alpha_{i}^{\prime}$ are the signed degree sequences of a signed $k$-partite graph, where $\alpha_{1}^{\prime}$ is obtained from $\alpha_{1}$ by deleting $d_{11}$ and $\alpha_{2}^{\prime}, \alpha_{3}^{\prime}, \cdots, \alpha_{k}^{\prime}$ are obtained from $\alpha_{2}, \alpha_{3}, \cdots, \alpha_{k}$ by reducing $r$ greatest entries of $\alpha_{2}, \alpha_{3}, \cdots, \alpha_{k}$ by 1 each and adding $s$ least entries of $\alpha_{2}, \alpha_{3}, \cdots, \alpha_{k}$ by 1 each. Proof. Let $r$ and $s$ be integers with $d_{11}=r-s$ and $\leq s \leq \frac{1}{2}\left(\sum_{j=2}^{k} n_{j}-d_{11}\right)$ such that $\alpha_{i}^{\prime}, 1 \leq i \leq k$, are the signed degree sequences of a signed $k$-partite graph $G^{\prime}\left(V_{1}^{\prime}\right.$, $\left.V_{2}^{\prime}, \prime, V_{k}^{\prime}\right)$.

Let $V_{1}^{\prime}=\left\{v_{12}, v_{13}, \cdots, v_{1 n_{1}}\right\}$ and $V_{i}^{\prime}=\left\{v_{i 1}, v_{i 2}, \cdots, v_{i n_{i}}\right\}, 2 \leq i \leq k$. Let $U$ be the set of $r$ vertices of $V_{2}^{\prime}, V_{3}^{\prime}, \cdots, V_{k}^{\prime}$ with highest signed degrees, $W$ be the set of $s$ vertices of $V_{2}^{\prime}, V_{3}^{\prime}, \cdots, V_{k}^{\prime}$ with least signed degrees and let $Z=\cup_{j=2}^{k} V_{j}^{\prime}-U-W$. Then a signed $k$-partite graph with signed degree sequences $\alpha_{i}, 1 \leq i \leq k$, can be obtained by adding a vertex $v_{11}$ to $V_{1}^{\prime}$ so that there are $r$ positive edges from $v_{11}$ to the vertices of $U$ and $s$ negative edges from $v_{11}$ to the vertices of $W$. Note that the signed degree of $v_{11}$ is $r-s=d_{11}$.

Conversely, let $\alpha_{i}, 1 \leq i \leq k$, be the signed degree sequences of a signed $k$-partite 
graph. Let the vertex sets of the signed $k$-partite graph be $V_{i}=\left\{v_{i 1}, v_{i 2}, \cdots, v_{i n_{i}}\right\}$ such that $\operatorname{sdeg}\left(v_{i j}\right)=d_{i j}, 1 \leq i \leq k, 1 \leq j \leq n_{i}$.

Among all the signed $k$-partite graphs with $\alpha_{i}, 1 \leq i \leq k$, as the signed degree sequences, let $G\left(V_{1}, V_{2}, \cdots, V_{k}\right)$ be one with the property that the sum $S$ of the signed degrees of the vertices of $V_{2}, V_{3}, \cdots, V_{k}$ joined to $v_{11}$ by positive edges is maximum. Let $d_{11}^{+}=r$ and $d_{11}^{-}=s$ be respectively the number of positive edges and the number of negative edges incident with $v_{11}$. Then $\operatorname{sdeg}\left(v_{11}\right)=d_{11}=d_{11}^{+}-d_{11}^{-}=r-s$ and $\operatorname{deg}\left(v_{11}\right)=d_{11}^{+}+d_{11}^{-}=r+s \leq \sum_{j=2}^{k} n_{j}$, and hence $0 \leq s \leq \frac{1}{2}\left(\sum_{j=2}^{k} n_{j}-d_{11}\right)$. Let $U$ be the set of $r$ vertices of $V_{2}, V_{3}, \cdots, V_{k}$ with highest signed degrees and let $W=\cup_{j=2}^{k} V_{j}-U$.

We claim that $v_{11}$ must be joined by positive edges to the vertices of $U$. If this is not true, then there exist vertices $v_{g h} \in U$ and $v_{m n} \in W$ such that the edge $v_{11} v_{m n}$ is positive and either (i) $v_{11} v_{g h}$ is a negative edge or (ii) $v_{11}$ and $v_{g h}$ are not adjacent in $G\left(V_{1}, V_{2}, \cdots, V_{k}\right)$. As $\operatorname{sdeg}\left(v_{g h}\right) \geq \operatorname{sdeg}\left(v_{m n}\right)$, that is $d_{g h} \geq d_{m n}$, therefore we consider only (i) and then (ii) is similar to (i).

We note that if there exists a vertex $v_{p q}\left(\neq v_{11}\right)$ such that $v_{p q} v_{g h}$ is a positive edge and $v_{p q} v_{m n}$ is a negative edge, then change the signs of these edges so that $v_{11} v_{g h}$ and $v_{p q} v_{m n}$ are positive, and $v_{11} v_{m n}$ and $v_{p q} v_{g h}$ are negative. Hence we obtain a signed $k$-partite graph with signed degree sequences $\alpha_{i}, 1 \leq i \leq k$, in which the sum of the signed degrees of the vertices of $V_{2}, V_{3}, \cdots, V_{k}$ joined to $v_{11}$ by positive edges exceeds $S$, a contradiction. So assume that no such vertex $v_{p q}$ exists.

Now, suppose that $v_{g h}$ is not incident to any positive edge. Since $\operatorname{sdeg}\left(v_{g h}\right) \geq$ $\operatorname{sdeg}\left(v_{m n}\right)$, that is $d_{g h} \geq d_{m n}$, then there exist at least two vertices $v_{p q}$ and $v_{l t}$ (both distinct from $v_{11}$ ) such that $v_{p q} v_{m n}$ and $v_{l t} v_{m n}$ are negative edges and both $v_{p q}$ and $v_{l t}$ are not adjacent to $v_{g h}$. Then by changing the edges so that $v_{11} v_{g h}$ is a positive edge, and $v_{11} v_{m n}, v_{g h} v_{p q}, v_{g h} v_{l t}$ are negative edges, we again get a contradiction. Hence $v_{g h}$ is incident to at least one positive edge.

We claim that there exists at least one vertex $v_{y z}$ such that $v_{y z} v_{g h}$ is a positive edge and $v_{y z}$ is not adjacent to $v_{m n}$. Suppose on contrary that whenever $v_{g h}$ is joined to a vertex by a positive edge, then $v_{m n}$ is also joined to this vertex by a positive edge. Since $\operatorname{sdeg}\left(v_{g h}\right) \geq \operatorname{sdeg}\left(v_{m n}\right)$, that is $d_{g h} \geq d_{m n}$, then again we have the same situation as above, from which we get a contradiction. Thus there exists a vertex $v_{y z}$ such that $v_{y z} v_{g h}$ is a positive edge and $v_{y z}$ is not adjacent to $v_{m n}$. Similarly, it can be shown that there exists a vertex $v_{p q}$ such that $v_{p q} v_{m n}$ is a negative edge and $v_{p q}$ is not adjacent to $v_{g h}$. By changing the edges so that $v_{11} v_{g h}, v_{m n} v_{y z}$ are positive edges, and $v_{11} v_{m n}, v_{g h} v_{p q}$ are negative edges, we again get a contradiction. Hence $v_{11}$ is joined by positive edges to the vertex of $U$.

In a similar way, it can be shown that $v_{11}$ is joined by negative edge to the $s$ vertices of $V_{2}, V_{3}, \cdots, V_{k}$ with least signed degrees.

Hence $G\left(V_{1}, V_{2}, \cdots, V_{k}\right)-v_{11}$ is a signed $k$-partite graph with $\alpha_{i}^{\prime}, 1 \leq i \leq k$, as the signed degree sequences.

Theorem 8 also provides an algorithm for determining whether or not the standard sequences $\alpha_{i}, 1 \leq i \leq k$, are the signed degree sequences, and for constructing a corresponding signed $k$-partite graph. Suppose $\alpha_{i}=\left[d_{i 1}, d_{i 2}, \cdots, d_{i n_{i}}\right], 1 \leq i \leq k$, be the standard signed degrees sequences of a signed k-partite graph with parts $V_{i}=\left\{v_{i 1}, v_{i 2}, \cdots, v_{i n_{i}}\right\}$. Let $d_{11}=r-s$ and $0 \leq s \leq \frac{1}{2}\left(\sum_{j=2}^{k} n_{j}-d_{11}\right)$. Deleting $d_{11}$ and reducing $r$ greatest entries of $\alpha_{2}, \alpha_{3}, \cdots, \alpha_{k}$ by 1 each and adding $s$ least entries of $\alpha_{2}, \alpha_{3}, \cdots, \alpha_{k}$ by 1 each to form $\alpha_{2}^{\prime}, \alpha_{3}^{\prime}, \cdots, \alpha_{k}^{\prime}$. Then edges are defined by $v_{11} v_{i j}^{+}$if $d_{i j}^{\prime} s$ are reduced by $1 ; v_{11} v_{1 j}^{-}$ if $d_{i j}^{\prime} s$ are increased by 1 , and $v_{11}$ and $v_{i j}$ are not adjacent if $d_{i j}^{\prime} s$ are unchanged. For $\alpha_{i}$, edges are defined by $v_{11} v_{i j}^{-}$if $d_{i j}^{\prime} s$ are reduced by $1 ; v_{11} v_{i j}^{+}$if $d_{i j}^{\prime} s$ are increased by 
1 , and $v_{11}$ and $v_{i j}$ are not adjacent if $d_{i j}^{\prime} s$ are unchanged. If the conditions of standard sequences do not hold, then we delete $d_{i 1}$ for that $i$ for which the conditions of standard sequences get satisfied. If this method is applied recursively, then a signed $k$-partite graph with signed degree sequences $\alpha_{i}, 1 \leq i \leq k$, is constructed.

\section{Signed degree sets in signed $k$-partite graphs}

Let $G\left(V_{1}, V_{2}, \cdots, V_{k}\right)$ be a signed $k$-partite graph with $X \subseteq V_{i}, Y \subseteq V_{j}(i \neq j)$. If each vertex of $X$ is joined to every vertex of $Y$ by a positive (negative) edge, then it is denoted by $X \oplus Y(X \ominus Y)$.

The set $S$ of distinct signed degrees of the vertices in a signed $k$-partite graph $G\left(V_{1}, V_{2}, \cdots, V_{k}\right)$ is called its signed degree set. Also, a signed $k$-partite graph $G\left(V_{1}, V_{2}, \cdots, V_{k}\right)$ is said to be connected if each vertex $v_{i} \in V_{i}$; is connected to every vertex $v_{j} \in V_{j}$.

The following result shows that every set of positive integers is a signed degree set of some connected signed $k$-partite graph.

Theorem 9. Let $d_{1}, d_{2}, \cdots, d_{t}$ be positive integers. Then there exists a connected signed $k$-partite graph with signed degree set

$$
S=\left\{d_{1}, \sum_{i=1}^{2} d_{i}, \cdots, \sum_{i=1}^{t} d_{i}\right\} .
$$

Proof. We consider the following two cases. (i) $k$ even, (ii) $k$ odd.

Case (i). Let $k=2 m$, where $m \geq 1$. Construct a signed $k$-partite graph $G\left(V_{1}, V_{2}, \cdots, V_{2 m}\right)$ as follows.

Let

$$
\begin{aligned}
& V_{1}=P_{1} \cup Q_{1} \cup R_{1} \cup S_{1} \cup X_{1} \cup X_{1}^{\prime} \cup X_{1}^{\prime \prime} \cup X_{2} \cup X_{2}^{\prime} \cup X_{2}^{\prime \prime} \cup \cdots \cup X_{t-1} \cup X_{t-1}^{\prime} \cup X_{t-1}^{\prime \prime}, \\
& V_{2}=P_{2} \cup Q_{2} \cup R_{2} \cup S_{2} \cup Y_{1} \cup Y_{1}^{\prime} \cup Y_{2} \cup Y_{2}^{\prime} \cup \cdots \cup Y_{t-1} \cup Y_{t-1}^{\prime}, \\
& V_{3}=P_{3} \cup Q_{3}, \\
& \vdots \\
& V_{2 m-1}=P_{2 m-1} \cup Q_{2 m-1}, \\
& V_{2 m}=P_{2 m} \cup Q_{2 m},
\end{aligned}
$$

where

(a) $P_{1}, Q_{1}, R_{1}, S_{1}, X_{1}, X_{1}^{\prime}, X_{1}^{\prime \prime}, X_{2}, X_{2}^{\prime}, X_{2}^{\prime \prime}, \cdots, X_{t-1}, X_{t-1}^{\prime}, X_{t-1}^{\prime \prime}$ are pairwise disjoint,

(b) $P_{2}, Q_{2}, R_{2}, S_{2}, Y_{1}, Y_{1}^{\prime}, Y_{2}, Y_{2}^{\prime}, \cdots, Y_{t-1}, Y_{t-1}^{\prime}$ are pairwise disjoint,

(c) For all $i, P_{i} \cap Q_{i}=\phi, 3 \leq i \leq 2 m$ and $\left|P_{i}\right|=\left|Q_{i}\right|=d_{1}, 1 \leq i \leq 2 m ;\left|R_{i}\right|=\left|S_{i}\right|=d_{1}$, $1 \leq i \leq 2 ;\left|X_{i}\right|=\left|X_{i}^{\prime}\right|=\left|Y_{i}\right|=\left|Y_{i}^{\prime}\right|=d_{1}, 1 \leq i \leq t-1 ;\left|X_{i}^{\prime \prime}\right|=d_{2}+d_{3}+\cdots+d_{i+1}$, $1 \leq i \leq t-1$.

For all $i$, let $P_{i} \oplus Q_{i+1}, 1 \leq i \leq 2 m-1 ; Q_{i} \oplus P_{i+1}, 1 \leq i \leq 2 m-1 ; Q_{1} \oplus R_{2}, R_{1} \oplus$ $Q_{2}, R_{1} \oplus S_{2}, S_{1} \oplus R_{2}, X_{1} \oplus S_{2}, X_{1}^{\prime} \oplus R_{2}, X_{i} \oplus Y_{i}^{\prime}, 1 \leq i \leq t-1 ; X_{i}^{\prime} \oplus Y_{i}, 1 \leq i \leq t-1 ;$ $X_{i}^{\prime \prime} \oplus Y_{i}^{\prime}, 1 \leq i \leq t-1 ; X_{i} \oplus Y_{i-1}^{\prime}, 2 \leq i \leq t-1 ; X_{i}^{\prime} \oplus Y_{i-1}, 2 \leq i \leq t-1$; for all even $i, P_{i} \ominus P_{i+1}, 2 \leq i \leq 2 m-2 ; Q_{i} \ominus Q_{i+1}, 2 \leq i \leq 2 m-2$; and for all $i, Q_{1} \ominus Q_{2}, R_{1} \ominus R_{2}, X_{1} \ominus R_{2}, X_{1}^{\prime} \ominus S_{2}, X_{i} \ominus Y_{i-1}, 2 \leq i \leq t-1 ; X_{i}^{\prime} \ominus Y_{i-1}^{\prime}$, $2 \leq i \leq t-1$.

Then the signed degrees of the vertices of $G\left(V_{1}, V_{2}, \cdots, V_{2 m}\right)$ are as follows. 
$\operatorname{sdeg}\left(p_{1}\right)=\left|Q_{2}\right|-0=d_{1}$ for all $p_{1} \in P_{1}$

for even $i, 2 \leq i \leq 2 m-2$

$\operatorname{sdeg}\left(p_{i}\right)=\left|Q_{i-1}\right|+\left|Q_{i+1}\right|-\left|P_{i+1}\right|=d_{1}+d_{1}-d_{1}=d_{1}$, for all $p_{i} \in P_{i}$

for odd $i, 3 \leq i \leq 2 m-1$

$\operatorname{sdeg}\left(p_{i}\right)=\left|Q_{i-1}\right|+\left|Q_{i+1}\right|-\left|P_{i-1}\right|=d_{1}+d_{1}-d_{1}=d_{1}$, for all $p_{i} \in P_{i}$,

$\operatorname{sdeg}\left(p_{2 m}\right)=\left|Q_{2 m-1}\right|-0=d_{1}$, for all $p_{2 m} \in P_{2 m}$;

$\operatorname{sdeg}\left(q_{1}\right)=\left|P_{2}\right|+\left|R_{2}\right|-\left|Q_{2}\right|=d_{1}+d_{1}-d_{1}=d_{1}$, for all $q_{1} \in Q_{1}$;

$\operatorname{sdeg}\left(q_{2}\right)=\left|P_{1}\right|+\left|R_{1}\right|+\left|P_{3}\right|-\left(\left|Q_{1}\right|+\left|Q_{3}\right|\right)=d_{1}+d_{1}+d_{1}-\left(d_{1}+d_{1}\right)=d_{1}$, for all $q_{2} \in Q_{2}$;

for odd $i, 3 \leq i \leq 2 m-1$

$\operatorname{sdeg}\left(q_{i}\right)=\left|P_{i-1}\right|+\left|P_{i+1}\right|-\left|Q_{i-1}\right|=d_{1}+d_{1}-d_{1}=d_{1}$, for all $q_{i} \in Q_{i}$;

for even $i, 4 \leq i \leq 2 m-2$

$\operatorname{sdeg}\left(q_{i}\right)=\left|{ }_{i-1}\right|+\left|P_{i+1}\right|-\left|Q_{i+1}\right|=d_{1}+d_{1}-d_{1}=d_{1}$, for all $q_{i} \in Q_{i}, \operatorname{sdeg}\left(q_{2 m}\right)=$ $\left|P_{2 m-1}\right|-0=d_{1}$, for all $q_{2 m} \in Q_{2 m}, \operatorname{sdeg}\left(r_{1}\right)=\left|Q_{2}\right|+\left|S_{2}\right|-\left|R_{2}\right|=d_{1}+d_{1}-d_{1}=d_{1}$, for all $r_{1} \in R_{1}$,

$\operatorname{sdeg}\left(s_{1}\right)=\left|R_{2}\right|-0=d_{1}$, for all $s_{1} \in S_{1}$,

$\operatorname{sdeg}\left(r_{2}\right)=\left|Q_{1}\right|+\left|S_{1}\right|+\left|X_{1}^{\prime}\right|-\left(\left|R_{1}\right|+\left|X_{1}\right|\right)=d_{1}+d_{1}+d_{1}-\left(d_{1}+d_{1}\right)=d_{1}$, for all $r_{2} \in R_{2}$,

$\operatorname{sdeg}\left(s_{2}\right)=\left|R_{1}\right|+\left|X_{1}\right|-\left|X_{1}^{\prime}\right|=d_{1}+d_{1}-d_{1}=d_{1}$, for all $s_{2} \in S_{2}$,

$\operatorname{sdeg}\left(x_{1}\right)=\left|S_{2}\right|+\left|Y_{1}^{\prime}\right|-\left|R_{2}\right|=d_{1}+d_{1}-d_{1}=d_{1}$, for all $x_{1} \in X_{1}$,

$\operatorname{sdeg}\left(x_{1}^{\prime}\right)=\left|R_{2}\right|+\left|Y_{1}\right|-\left|S_{2}\right|=d_{1}+d_{1}-d_{1}=d_{1}$, for all $x_{1}^{\prime} \in X_{1}^{\prime}$,

$\operatorname{sdeg}\left(x_{1}^{\prime \prime}\right)=\left|Y_{1}^{\prime}\right|-0=d_{1}$, for all $x_{1}^{\prime \prime} \in X_{1}^{\prime \prime}$;

for $2 \leq i \leq t-1$

$\operatorname{sdeg}\left(x_{i}\right)=\left|Y_{i-1}^{\prime}\right|+\left|Y_{i}^{\prime}\right|-\left|Y_{i-1}\right|=d_{1}+d_{1}-d_{1}=d_{1}$, for all $x_{i} \in X_{i}$;

for $2 \leq i \leq t-1$

$\operatorname{sdeg}\left(x_{i}^{\prime}\right)=\left|Y_{i-1}\right|+\left|Y_{i}\right|-\left|Y_{i-1}^{\prime}\right|=d_{1}+d_{1}-d_{1}=d_{1}$, for all $x_{i}^{\prime} \in X_{i}^{\prime}$;

for $2 \leq i \leq t-1$

$\operatorname{sdeg}\left(\bar{x}_{i}^{\prime \prime}\right)=\left|Y_{i}^{\prime}\right|-0=d_{1}$, for all $x_{i}^{\prime \prime} \in X_{i}^{\prime \prime}$;

for $1 \leq i \leq t-2$

$\operatorname{sdeg}\left(y_{i}\right)=\left|X_{i}^{\prime}\right|+\left|X_{i+1}^{\prime}\right|-\left|X_{i+1}\right|=d_{1}+d_{1}-d_{1}=d_{1}$, for all $y_{i} \in Y_{i}$

$\operatorname{sdeg}\left(y_{t-1}\right)=\left|X_{t-1}^{\prime}\right|-0=d_{1}$, for all $y_{t-1} \in Y_{t-1}$;

for $1 \leq i \leq t-2$

$\operatorname{sdeg}\left(\overline{y_{i}^{\prime}}\right)=\left|X_{i}\right|+\left|X_{i}^{\prime \prime}\right|+\left|X_{i+1}\right|-\left|X_{i+1}^{\prime}\right|=d_{1}+d_{2}+d_{3}+\cdots+d_{i+1}+d_{1}-d_{1}=\sum_{j=1}^{i+1} d_{j}$, for all $y_{i}^{\prime} \in Y_{i}^{\prime}$,

and $\operatorname{sdeg}\left(y_{t-1}^{\prime}\right)=\left|X_{t-1}\right|+\left|X_{t-1}^{\prime \prime}\right|=d_{1}+d_{2}+d_{3}+\cdots+d_{t}=\sum_{j=1}^{t} d_{j}$, for all $y_{t-1}^{\prime} \in Y_{t-1}^{\prime}$. Therefore signed degree set of $G\left(V_{1}, V_{2}, \prime, V_{2 m}\right)$ is $S=\left\{d_{1}, \sum_{i=1}^{2} d_{i}, \prime, \sum_{i=1}^{t} d_{i}\right\}$.

Case (ii). Let $k=2 m+1$, where $m \geq 1$. This follows by using the construction as in case (i), and taking another partite set $V_{2 m+1}=P_{2 m+1} \cup Q_{2 m+1}$ with $P_{2 m+1} \cap Q_{2 m+1}=$ $\phi,\left|P_{2 m+1}\right|=\left|Q_{2 m+1}\right|=d_{1}, P_{2 m} \oplus Q_{2 m+1}, Q_{2 m} \oplus P_{2 m+1}, P_{2 m+1} \oplus P_{1}, P_{2 m+1} \oplus R_{2}, Q_{2 m+1} \oplus$ $S_{1}, Q_{1} \oplus S_{2}$ and $P_{2 m} \ominus P_{2 m+1}, Q_{2 m} \ominus Q_{2 m+1}, P_{2 m+1} \ominus Q_{1}, P_{1} \ominus R_{2}, S_{1} \ominus S_{2}$.

Clearly, by construction, the above signed $k$-partite graphs are connected. Hence the result follows.

By interchanging positive edges with negative edges in Theorem 9, we obtain the following result.

Corollary 10. Every set of negative integers is a signed degree set of some connected signed $k$-partite graph. 
Finally, we have the following result.

Theorem 11. Every set of integers is a signed degree set of some connected signed $k$-partite graph.

Proof. Let $S$ be a set of integers. Then we have the following five cases.

Case (i). $S$ is a set of positive (negative) integers. Then the result follows by Theorem 9 (Corollary 10).

Case (ii). $S=\{0\}$. Then a signed $k$-partite graph $G\left(V_{1}, V_{2}, \cdots, V_{k}\right)$ with $V_{i}=\left\{v_{i}, v_{i}^{\prime}\right\}$ for all $i, 1 \leq i \leq k$, in which $v_{i} v_{i+1}^{\prime}, v_{i}^{\prime} v_{i+1}$ for all $i, 1 \leq i \leq k-1$, are positive edges and $v_{i} v_{i+1}, v_{i}^{\prime} v_{i+1}^{\prime}$ for all $i, 1 \leq i \leq k-1$, are negative edges has signed degree set $S$.

Case (iii). $S$ is a set of non-negative (non-positive) integers. Let $S=S^{\prime} \cup\{0\}$, where $S^{\prime}$ be a set of positive(negative) integers. Then by Theorem 9 (Corollary 10), there is a connected signed $k$-partite graph $G^{\prime}\left(V_{1}^{\prime}, V_{2}^{\prime}, \cdots, V_{k}^{\prime}\right)$ with signed degree set $S^{\prime}$. Construct a new signed $k$-partite graph $G\left(V_{1}, V_{2}, \cdots, V_{k}\right)$ as follows.

Let $V_{1}=V_{1}^{\prime} \cup\left\{x_{1}\right\} \cup\left\{y_{1}\right\}, V_{2}=V_{2}^{\prime} \cup\left\{x_{2}\right\} \cup\left\{y_{2}\right\}, V_{3}=V_{3}^{\prime}, \cdots, V_{k}=V_{k}^{\prime}$, with $V_{1}^{\prime} \cap\left\{x_{1}\right\}=\phi, V_{1}^{\prime} \cap\left\{y_{1}\right\}=\phi,\left\{x_{1}\right\} \cap\left\{y_{1}\right\}=\phi, V_{2}^{\prime} \cap\left\{x_{2}\right\}=\phi, V_{2}^{\prime} \cap\left\{y_{2}\right\}=\phi,\left\{x_{2}\right\} \cap\left\{y_{2}\right\}=\phi$. Let $v_{1}^{\prime} x_{2}, x_{1} v_{2}^{\prime}, y_{1} y_{2}$ be positive edges, $v_{1}^{\prime} y_{2}, x_{1} x_{2}, y_{1} v_{2}^{\prime}$ be negative edges, where $v_{1}^{\prime} \in$ $V_{1}^{\prime}, v_{2}^{\prime} \in V_{2}^{\prime}$ and let there be all the edges of $G^{\prime}\left(V_{1}^{\prime}, V_{2}^{\prime}, \cdots, V_{k}^{\prime}\right)$. Then $G\left(V_{1}, V_{2}, \cdots, V_{k}\right)$ has signed degree set $S$. We note that addition of the positive edges $v_{1}^{\prime} x_{2}, x_{1} v_{2}^{\prime}, y_{1} y_{2}$ and negative edges $v_{1}^{\prime} y_{2}, x_{1} x_{2}, y_{1} v_{2}^{\prime}$ do not effect the signed degrees of the vertices of $G^{\prime}\left(V_{1}^{\prime}, V_{2}^{\prime}, \cdots, V_{k}^{\prime}\right)$, and the vertices $x_{1}, y_{1}, x_{2}, y_{2}$ have signed degrees zero each.

Case (iv). $S$ is a set of non-zero integers. Let $S=S^{\prime} \cup S^{\prime \prime}$, where $S^{\prime}$ and $S^{\prime \prime}$ are sets of positive and negative integers respectively. Then by Theorem 9 (Corollary 10), there are connected signed $k$-partite graphs $G^{\prime}\left(V_{1}^{\prime}, V_{2}^{\prime}, \cdots, V_{k}^{\prime}\right)$ and $G^{\prime \prime}\left(V_{1}^{\prime \prime}, V_{2}^{\prime \prime}, \cdots, V_{k}^{\prime \prime}\right)$ with signed degree sets $S^{\prime}$ and $S^{\prime \prime}$ respectively. Suppose $G_{1}^{\prime}\left(V_{11}^{\prime}, V_{21}^{\prime}, \cdots, V_{k 1}^{\prime}\right)$ and $G_{2}^{\prime \prime}\left(V_{12}^{\prime \prime}, V_{22}^{\prime \prime}, \cdots, V_{k 2}^{\prime \prime}\right)$ are the copies of $G^{\prime}\left(V_{1}^{\prime}, V_{2}^{\prime}, \cdots, V_{k}^{\prime}\right)$ and $G^{\prime \prime}\left(V_{1}^{\prime \prime}, V_{2}^{\prime \prime}, \cdots, V_{k}^{\prime \prime}\right)$ with signed degree sets $S^{\prime}$ and $S^{\prime \prime}$ respectively. Construct a new signed $k$-partite graph $G\left(V_{1}, V_{2}, \cdots, V_{k}\right)$ as follows.

Let

$$
\begin{aligned}
& V_{1}=V_{1}^{\prime} \cup V_{11}^{\prime} \cup V_{1}^{\prime \prime} \cup V_{12}^{\prime \prime}, \\
& V_{2}=V_{2}^{\prime} \cup V_{21}^{\prime} \cup V_{2}^{\prime \prime} \cup V_{22}^{\prime \prime}, \\
& V_{3}=V_{3}^{\prime} \cup V_{31}^{\prime} \cup V_{3}^{\prime \prime} \cup V_{32}^{\prime \prime}, \\
& \vdots \\
& V_{k}=V_{k}^{\prime} \cup V_{k 1}^{\prime} \cup V_{k}^{\prime \prime} \cup V_{k 2}^{\prime \prime},
\end{aligned}
$$

with $V_{i}^{\prime} \cap V_{i 1}^{\prime}=\phi, V_{i}^{\prime} \cap V_{i}^{\prime \prime}=\phi, V_{i}^{\prime} \cap V_{i 2}^{\prime \prime}=\phi, V_{i 1}^{\prime} \cap V_{i}^{\prime \prime}=\phi, V_{i 1}^{\prime} \cap V_{i 2}^{\prime \prime}=\phi, V_{i}^{\prime \prime} \cap V_{i 2}^{\prime \prime}=\phi$. Let $v_{1}^{\prime} v_{22}^{\prime \prime}, v_{11}^{\prime} v_{2}^{\prime \prime}$ be positive edges, $v_{1}^{\prime} v_{2}^{\prime}, v_{11}^{\prime} v_{22}^{\prime \prime}$ be negative edges, where $v_{1}^{\prime} \in V_{1}^{\prime}, v_{11}^{\prime} \in$ $V_{11}^{\prime}, v_{2}^{\prime \prime} \in V_{2}^{\prime \prime}, v_{22}^{\prime \prime} \in V_{22}^{\prime \prime}$ and let there be all the edges of

$G^{\prime}\left(V_{1}^{\prime}, V_{2}^{\prime}, \cdots, V_{k}^{\prime}\right), G_{1}^{\prime}\left(V_{11}^{\prime}, V_{21}^{\prime}, \cdots, V_{k 1}^{\prime}\right), G^{\prime \prime}\left(V_{1}^{\prime \prime}, V_{2}^{\prime \prime}, \cdots, V_{k}^{\prime \prime}\right)$ and $G_{2}^{\prime \prime}\left(V_{12}^{\prime \prime}, V_{22}^{\prime \prime}, \cdots, V_{k 2}^{\prime \prime}\right)$. Then $G\left(V_{1}, V_{2}, \cdots, V_{k}\right)$ has signed degree set $S$.

We note that addition of the positive edges $v_{1}^{\prime} v_{22}^{\prime \prime}, v_{11}^{\prime} v_{2}^{\prime \prime}$ and negative edges $v_{1}^{\prime} v_{2}^{\prime \prime}, v_{11}^{\prime} v_{22}^{\prime \prime}$ do not effect the signed degrees of the vertices of $G^{\prime}\left(V_{1}^{\prime}, V_{2}^{\prime}, \cdots, V_{k}^{\prime}\right), G_{1}^{\prime}\left(V_{11}^{\prime}, V_{21}^{\prime}, \cdots, V_{k 1}^{\prime}\right)$, $G^{\prime \prime}\left(V_{1}^{\prime \prime}, V_{2}^{\prime \prime}, \cdots, V_{k}^{\prime \prime}\right)$ and $G_{2}^{\prime \prime}\left(V_{12}^{\prime \prime}, V_{22}^{\prime \prime}, \cdots, V_{k 2}^{\prime \prime}\right)$.

Case (v). $S$ is the set of all integers. Let $S=S^{\prime} \cup S^{\prime \prime} \cup\{0\}$, where $S^{\prime}$ and $S^{\prime \prime}$ are sets of positive and negative integers respectively. Then by Theorem 9 (Corollary 10), there exist connected signed $k$-partite graphs $G^{\prime}\left(V_{1}^{\prime}, V_{2}^{\prime}, \cdots, V_{k}^{\prime}\right)$ and $G^{\prime \prime}\left(V_{1}^{\prime \prime}, V_{2}^{\prime \prime}, \cdots, V_{k}^{\prime \prime}\right)$ with signed degree sets $S^{\prime}$ and $S^{\prime \prime}$ respectively. Construct a new signed $k$-partite graph $G\left(V_{1}, V_{2}, \cdots, V_{k}\right)$ as follows. 
Let

$$
\begin{aligned}
& V_{1}=V_{1}^{\prime} \cup V_{1}^{\prime \prime} \cup\{x\}, \\
& V_{2}=V_{2}^{\prime} \cup V_{2}^{\prime \prime} \cup\{y\}, \\
& V_{3}=V_{3}^{\prime} \cup V_{3}^{\prime \prime}, \\
& \vdots \\
& V_{k}=V_{k}^{\prime} \cup V_{k}^{\prime \prime},
\end{aligned}
$$

with $V_{i}^{\prime} \cap V_{i}^{\prime \prime}=\phi, V_{1}^{\prime} \cap\{x\}=\phi, V_{1}^{\prime \prime} \cap\{x\}=\phi, V_{2}^{\prime} \cap\{y\}=\phi, V_{2}^{\prime \prime} \cap\{y\}=\phi$. Let $v_{1}^{\prime} v_{2}^{\prime \prime}, v_{1}^{\prime \prime} y, x v_{2}^{\prime}$ be positive edges, $v_{1}^{\prime} y, v_{1}^{\prime \prime} v_{2}^{\prime}, x v_{2}^{\prime \prime}$ be negative edges, where $v_{1}^{\prime} \in V_{1}^{\prime}, v_{1}^{\prime \prime} \in$ $V_{1}^{\prime \prime}, v_{2}^{\prime} \in V_{2}^{\prime}, v_{2}^{\prime \prime} \in V_{2}^{\prime \prime}$, and let there be all the edges of $G^{\prime}\left(V_{1}^{\prime}, V_{2}^{\prime}, \cdots, V_{k}^{\prime}\right)$ and $G^{\prime \prime}\left(V_{1}^{\prime \prime}, V_{2}^{\prime \prime}, \cdots, V_{k}^{\prime \prime}\right)$. Therefore $G\left(V_{1}, V_{2}, \cdots, V_{k}\right)$ has signed degree set $S$. We note that addition of the positive edges $v_{1}^{\prime} v_{2}^{\prime \prime}, v_{1}^{\prime \prime} y, x v_{2}^{\prime}$ and negative edges $v_{1}^{\prime} y, v_{1}^{\prime \prime} v_{2}^{\prime}, x v_{2}^{\prime \prime}$ do not effect the signed degrees of the vertices of $G^{\prime}\left(V_{1}^{\prime}, V_{2}^{\prime}, \cdots, V_{k}^{\prime}\right)$ and $G^{\prime \prime}\left(V_{1}^{\prime \prime}, V_{2}^{\prime \prime}, \cdots, V_{k}^{\prime \prime}\right)$, and the vertices $\mathrm{x}$ and $\mathrm{y}$ have signed degrees zero each.

Clearly, by construction, all the signed $k$-partite graphs are connected. This proves the result.

\section{References}

[1 ] G. Charttrand, H. Gavlas, F. Harary, M. Schultz, On signed degrees in signed graphs, Czech. Math. J., 44 (1994) 677-690.

[2 ] S. L. Hakimi, On the realizability of a set of integers as degrees of the vertices of a graph, SIAM J. Appl. Math., 10 (1962) 496-506.

[3 ] F. Harary, On the notion of balance in a signed graph, Michigan Math. J., 2(1953) 143-146.

[4] D. Hoffman, H. Jordan, Signed graph factors and degree sequences, J. Graph Theory, 52 (2006) 27-36.

[5 ] H. Jordan, R. McBride, S. Tipnis, The convex hull of degree sequences of signed graphs, Discrete Math., 309 (2009) 5841-5848.

[6 ] S. Pirzada, T. A. Naikoo, F. A. Dar, Signed degree sets in signed graphs, Czech. Math. J., 57, 3 (2007) 843-848.

[7 ] S. Pirzada, T. A. Naikoo, F. A. Dar, A note on signed degree sets in signed bipartite graphs, Applicable Analysis and Discrete Math., 2, 1 (2008) 114-117.

[8 ] S. Pirzada, T. A. Naikoo, S. Pirzada, Signed degree sequences in signed bipartite graphs, AKCE International J. Graphs and Comb., 4, 2 (2007) 1-12.

[9 ] S. Pirzada, Signed degree sequences in signed graphs, Journal of Combinatorics, Information and System Sciences, 37, 2-4 (2012) 333-358.

[10 ] S. Pirzada, F. A. Dar, Signed degree sequences in signed tripartite graphs, J. Korean Society of Industrial and Applied Mathematics, 11, 2 (2007) 9-14.

[11 ] S. Pirzada, F. A. Dar, Signed degree sets in signed tripartite graphs, Math. Vesnik, 59 (2007) 121-124.

[12 ] J. H. Yan, K. W. Lie, D. Kuo, G. J. Chang, Signed degree sequences of signed graphs, J. Graph Theory, 26 (1997) 111-117. 